\title{
The Challenge in Treating the Elderly Colorectal Cancer Patient
}

\author{
Kirk A. Ludwig, MD \\ Department of Surgery, Medical College of Wisconsin, Milwaukee, WI
}

The population of the USA is aging and, by 2030, $19 \%$ of our population, or about 72.1 million, will be aged 65 years or older: more than twice their number in the year 2000. ${ }^{1}$ Treating the elderly colorectal cancer patient is becoming increasingly commonplace, and since surgery forms the basis of treatment, we find ourselves on the front line, maneuvering around the elderly patient's comorbidities, trying to avoid early morbidity and mortality, while at the same time balancing respect for the geriatric patient's concerns over quality-of-life issues and desire for reasonable short-term outcomes with the desire to achieve good long-term cancer control. The study by Dekker et al. adds to our understanding of what happens when we operate on elderly colorectal cancer patients. ${ }^{2}$ These data should help as we struggle with managing this disease in this patient population.

The primary conclusion put forth by the authors of this population-based study of colorectal cancer patients from the western part of The Netherlands is that "elderly colorectal cancer patients that survive the first year have the same survival as younger patients... with decreased survival in the elderly mainly due to differences in early mortality... implying that treating early stage colorectal cancer patients should focus on the peri-operative process and the first postoperative year." 2

A number of other studies also suggest that, for elderly patients in particular, the first postoperative year is very important. In a study utilizing the California Cancer Registry, patients 80 years or older had high in-hospital mortality and 1-year mortality, with medical complications, increasing comorbidities, and cancer stage being predictive factors. ${ }^{3}$ In another study examining over 42,000 colorectal cancer patients in the Surveillance,

(C) Society of Surgical Oncology 2011

Published Online: 24 March 2011

K. A. Ludwig, MD

e-mail: kludwig@mcw.edu
Epidemiology, and End Results (SEER)-Medicare database, the 30 -day readmission rate was $11 \%$, with a predicted probability of 1-year mortality of $16 \%$ for readmitted patients compared with $7 \%$ for those not readmitted. The most common causes of rehospitalization were ileus/ obstruction and infection. Significant predictors of readmission included male gender, comorbidity, emergent admission, prolonged hospital stay, blood transfusion, ostomy, and discharge to nursing home. The conclusion was that readmission to the hospital after colectomy for cancer predicted 1-year mortality. ${ }^{4}$ In a systematic review of surgery for colorectal cancer in elderly patients, the primary finding was that overall survival was reduced for elderly patients. ${ }^{5}$ However, for cancer-specific survival, age-related differences were much less striking, suggesting that the difference in overall survival between elderly and younger patients cannot be explained in terms of cancer deaths. Competing causes of mortality are probably the main cause of the decreased long-term survival in elderly patients with colorectal cancer. In addition, elderly patients were less likely than younger patients to undergo surgery for their colorectal cancer, the operation was less likely to be curative, and the likelihood of the surgery being elective as compared with emergent was less. A study from Germany examined age as a risk factor on the early postoperative results of surgery for colorectal cancer and found that, while there were no specific differences in the type of complications after surgery in those patients less than or older than age 80 years, the rates were significantly higher for the older patients and the older patients had a significantly higher postoperative mortality rate. This increase in postoperative morbidity and mortality rates associated with aging were particularly a result of postoperative pneumonia and cardiovascular complications. ${ }^{6}$

As surgeons, what can we do to make an impact in advising and treating our elderly colorectal cancer patients? Firstly, we must inform them that, with increasing age, comes an increased complication rate and an increased 
perioperative mortality rate. We must be hopeful but honest, so that our patients and their families have a proper appreciation of what is being undertaken. In the elective setting, we must be good at the basics of taking a history and physical, assessing comorbidities, and then optimizing organ system function with the help of our medical and anesthesia colleagues. Time spent in preparation is generally time well spent. We should not be rushing to the operating room. When the time comes to operate, we should make use of technologies that are specifically designed to decrease the physiologic impact of surgery: minimally invasive techniques. In a setting where the surgeon would like to absolutely minimize postoperative pain, surgical-site infection rates, and pulmonary and gastrointestinal tract dysfunction, and get a patient up and moving as quickly as possible, laparoscopic resection would seem to be the answer. ${ }^{7,8}$ However, recent data suggest that utilization rates of laparoscopic techniques for colorectal cancer resection remain less than $10 \%{ }^{8} \mathrm{We}$ should be doing better than that for our patients. Also, we must take to heart the concept that the consequences of our complications that occur early after surgery extend well past the 30 days generally thought of as the "perioperative" period. If we can get our patients through the early period without issues, they have a better chance of making it through their first postoperative year, giving them the same cancer-related survival as younger patients. Our mindset for treating the elderly may simply have to be different. They might need their own modified patient care path.

Finally, management of the elderly colorectal cancer patient requires use of the proper combination of surgical science and art. We must understand what can be accomplished with our various treatments, what the physiologic cost will be for the patient, and what each patient wants and can tolerate. Consideration of these various factors allows recommendation of a reasonable treatment approach. We face these issues on a daily basis. During a recent cancer center clinic, on a single day, I had three new rectal cancer patients, two in their late $80 \mathrm{~s}$ and one in his early $90 \mathrm{~s}$. Each was a World War II veteran living independently but with major medical issues. Each understood that, while long-term cancer survival was an interesting concept, each patient had as their major focus maintaining independence, maximizing quality of life, and minimizing pain and suffering related to both treatment and the cancer. Bending the rules regarding stage-specific treatment for their cancer to balance what they thought they could endure with what might be best in terms of standard rectal cancer treatment were compromises each was ready to accept. Each chose a relatively nonstandard treatment approach, different from what I would have done for a 55-year-old patient, each had the cancer treated, each survived the holy "30-day" perioperative period, and it looks like each will make it through the first year without a treatment-related significant morbidity or mortality. Thankfully, goal number one has been accomplished.

\section{REFERENCES}

1. Unites States Department of Health and Human Services, Administration on Aging. http://www.aoa.gov/AoARoot/Aging_Statistics/ index.aspx.

2. Dekker JWT, van den Broek CBM, Bastiaannet E, van de Geest LGM, Tollenaar RAEM, Liefers GJ. Importance of the first postoperative year in the prognosis of elderly colorectal cancer patients. Ann Surg Oncol. 2011. Doi:10.1245/s10434-011-1671-x.

3. Kunitake H, Zingmond DS, Ryoo J, Ko CY. Caring for octogenarian and nonagenarian patients with colorectal cancer: what should our standards and expectations be? Dis Colon Rectum. 2010;53:735-43.

4. Greenblatt DY, Weber SM, O'Connor ES, LoConte NK, Liou J, Smith MA. Readmission after colectomy for cancer predicts oneyear mortality. Ann Surg. 2010;251(4):659-69.

5. Colorectal Cancer Collaborative Group. Surgery for colorectal cancer in elderly patients: a systematic review. Lancet. 2000;356: 968-74.

6. Marusch F, Koch A, Schmidt U, Steinert R, Ueberrueck T, Bittner $\mathrm{R}$, Berg E, et al. The impact of the risk factor "age" on the early postoperative results of surgery for colorectal carcinoma and its significance for perioperative management. World J Surg. 2005; 29(8):1013-22.

7. Kiran RP, El-Gazzaz GH, Vogel JD, Remzi FH. Laparoscopic approach significantly reduces surgical site infections after colorectal surgery: data from the national surgical quality improvement program. J Am Coll Surg. 2010;211(2):232-8.

8. Robinson CN, Chen J, Balentine CJ, Sansgiry S, Marshall CL, Anaya DA, et al. Minimally invasive surgery is underutilized for colon cancer. Ann Surg Oncol. Published on line 07 January 2011. Doi:10.1245/s10434-010-1479-0. 